Published in final edited form as:

Clin Biomech (Bristol, Avon). 2018 June ; 55: 40-44. doi:10.1016/j.clinbiomech.2018.04.011.

\title{
Effects of Localized Vibration on Knee Joint Position Sense in Individuals with Anterior Cruciate Ligament Reconstruction
}

\author{
Takashi Nagai, ATC, $\mathbf{P h D}^{1,2}$, Nathaniel A. Bates, $\mathbf{P h D}^{1,2,3}$, Timothy E. Hewett, $\mathbf{P h D}^{1,2,3,4,5}$, \\ and Nathan D. Schilaty, DC, PhD ${ }^{1,2,3, \dagger}$ \\ ${ }^{1}$ Biomechanics Laboratories, Department of Orthopedic Surgery, Mayo Clinic, Rochester, \\ Minnesota, United States \\ ${ }^{2}$ Sports Medicine Center, Mayo Clinic, Rochester, Minnesota, United States \\ ${ }^{3}$ Department of Physiology \& Biomedical Engineering, Mayo Clinic, Rochester, Minnesota, United \\ States \\ ${ }^{4}$ Department of Physical Medicine \& Rehabilitation, Mayo Clinic, Rochester, Minnesota, United \\ States \\ ${ }^{5}$ Department of Biomedical Engineering, The Ohio State University, Columbus, Ohio, United \\ States
}

\begin{abstract}
Background-Anterior cruciate ligament injury can disrupt one's mechanoreceptors and result in decreased proprioception such as joint position sense and ultimately altered motor function. The application of localized vibration has been used to investigate the integrity of the sensorimotor system and the mechanisms of quadriceps function after anterior cruciate ligament injury and reconstruction. The purpose of the study is to evaluate joint position sense with and without vibration and compare among anterior cruciate ligament reconstructed, contralateral, and control limbs.
\end{abstract}

\begin{abstract}
Methods-Fourteen subjects with anterior cruciate ligament reconstruction ( 8 males and 6 females) and fourteen control subjects ( 7 males and 7 females) participated in the study. Subjects sat on an isokinetic dynamometer chair with localized vibration strapped on the quadriceps tendon while visual and auditory cues were removed. Subjects were asked to remember an active target position and replicate that position actively. The absolute difference between the target and replicated trial was used as joint position sense. There were three trials at three target positions $(15,45$, and 75 degrees of knee flexion) with and without vibration. The order of testing conditions was randomized. One-way analysis of variance or non-parametric equivalent (KruskalWallis test) was used to compare among limbs. Significance was set at $P<0.05$ a priori.
\end{abstract}

\footnotetext{
${ }^{\dagger}$ CORRESPONDING AUTHOR: Name: Nathan Schilaty, DC, PhD, Address: Biomechanics Laboratories - 200 First Street SW, Rochester, MN 55905, United States, schilaty.nathan@ mayo.edu.

Conflict of Interest Statement: None.

Publisher's Disclaimer: This is a PDF file of an unedited manuscript that has been accepted for publication. As a service to our customers we are providing this early version of the manuscript. The manuscript will undergo copyediting, typesetting, and review of the resulting proof before it is published in its final citable form. Please note that during the production process errors may be discovered which could affect the content, and all legal disclaimers that apply to the journal pertain.
} 
Findings-There were no significant joint position sense differences among anterior cruciate ligament reconstructed, contralateral, and control limbs with or without vibration $(p=0.207-$ $0.914)$.

Interpretation-There are several potential reasons for the current findings: vibration-induced post effect, locations of vibration, types of vibration, and rehabilitation status. Future studies should expand the current investigation and explore both sensory and motor functions in anterior cruciate ligament reconstructed subjects.

\section{Keywords}

proprioception; ACL; ACL-reconstruction; joint position sense; vibration; muscle spindles

\section{Introduction}

Anterior cruciate ligament (ACL) injury is a devastating musculoskeletal condition that takes, at minimum, several months for athletes to return to sport and can lead to long-term disability due to early progression of osteoarthritis (Ruano et al., 2017). The ACL provides mechanical restraint against the anterior tibial translational forces as well as valgus and internal rotation moments at the knee near an extended position (Bates et al., 2017; Butler et al., 1980; Nesbitt et al., 2014); therefore, ACL injury often occurs when those forces and moments exceed the ultimate strength of the ligament. In addition to the mechanical role of the ACL, it also contains four types of sensory receptors (mechanoreceptors): Ruffini endings (slow adapting receptors), Pacinian corpuscles (fast adapting receptors), Golgi tendon-like organs (slow adapting inhibitory receptors), and free nerve endings (pain receptors) (Freeman \& Wyke, 1967).

Collectively, along with mechanoreceptors from other sources (cutaneous, musculotendinous, and articular), mechanoreceptors in the ACL deliver proprioceptive information to the central nervous system (CNS), contribute to perception of joint position sense (JPS), indicate movement or kinesthesia (commonly evaluated using threshold to detect passive motion (TTDPM)), and influence motor responses via ligament-muscular reflex and muscle spindles and whole-body movements (Johansson et al., 1991). Accordingly, a recent systematic review concluded that knee proprioception is diminished after ACL injury because the mechanoreceptors are disrupted by injury (Relph et al., 2014). In turn, balance deficits are commonly reported in individuals with ACL injury, purportedly for the same reason (Lehmann et al., 2017).

Sensory disruption due to ACL injury is not likely fully restored even after ACL reconstruction surgery (ACLR), and concerted effort is likely required to compensate the loss of the ACL mechanoreceptors during rehabilitation (Nagelli \& Hewett, 2017). ACLR grafts undergo biological steps toward revascularization and ultimately resemble the native ACL in a process referred to as 'ligamentization' (Claes et al., 2011). In humans, this ligamentization process would likely depend on many factors (individual demographics, surgical techniques, graft types, rehabilitation, angiogenesis, etc.). The microstructures of the reconstructed graft may take two years to resemble a normal ACL (Zaffagnini et al., 2007). However, it is mostly unknown exactly when (if any) the ACL mechanoreceptors 
return to the functional level after the reconstruction (Kim et al., 2012; Young et al., 2016). Electrical stimulation with electromyography (EMG) on the hamstring muscles or JPS or TTDPM at the knee joint or is commonly used as a surrogate method of sensory function (Bonfim et al., 2003; Iwasa et al., 2000; Krogsgaard et al., 2011; MacDonald et al., 1996; Ochi et al., 2002; Ochi et al., 1999; Roberts et al., 2000). However, due to a limitation of non-physiological electrical stimulation used and limitations of proprioception tests (inability to isolate one single structure such as the ACL), it is difficult to examine the true sensory function of ACLR subjects.

To address the limitations of proprioception testing, application of high-frequency vibration to activate muscle spindles has been used to investigate the role of muscle spindles, proprioception senses (JPS and TTDPM), and sensorimotor function in the past (Goodwin et al., 1972; Proske \& Gandevia, 2012). In the ACL literature, vibration to the infrapatellar tendon has been used to investigate the integrity of the gamma-muscle spindle system and elucidate the mechanisms of quadriceps function after ACL injury and ACLR (Konishi et al., 2002; Konishi, 2011). After the application of vibration, knee extension strength in a healthy, uninjured group was significantly decreased along with reduced EMG activities of the vastus lateralis and vastus medialis while the strength and EMG in the ACLR group were unchanged (Konishi et al., 2002). A similar study found knee strength reduction in the contralateral leg while the knee strength was increased in the ACLR limb within the individuals with ACLR (Konishi, 2011). However, those studies did not investigate the effects of vibration on proprioception. Because JPS is the most common and easiest proprioception test to administer in a clinical setting, it could potentially be used to evaluate the integrity of the gamma-muscle spindle system in individuals with ACLR.

Therefore, the purposes of the current study were to examine the effects of vibration on JPS and to compare the patterns of changes in JPS between limbs in individuals with ACLR and between ACLR limb and external control limb. Based on the previous studies on JPS after ACLR and the effects on vibration on strength and EMG in the ACLR individuals and uninjured controls, it was hypothesized that JPS would improve in the ACLR limb and worsen in the contralateral and control limbs after the application of vibration, and the pattern of changes would be significantly different between limbs within the ACLR individuals and between the groups. It is clinically important to examine the gamma-muscle spindle system in the ACL injured and ACLR knee because any deficits should be addressed with treatment plans to assist athletes' recovery to return to sport. An absence of full recovery in both sensory and motor systems could jeopardize the reconstructed knee as well as the other knee for secondary injury.

\section{Methods}

\subsection{Participants}

Twenty-eight subjects ( 15 males and 13 females) participated in the study. A priori power analysis based on a previous study (Li et al., 2008) that examined the effects of vibration on JPS revealed that at least 14 subjects in each group were needed to achieve a power of 0.80 , alpha of 0.05 , and effect size of 0.71. Therefore, 14 individuals with ACLR (ACLR group) and 14 individuals in the control group were analyzed in this study. Those 14 individuals 
with ACLR ( 8 males and 6 females) had to be cleared for full participation by their orthopedic surgeons. The average time from the surgery to laboratory testing date was 298 days. Those 14 individuals ( 7 males and 7 females) in the control group had to have no prior history of the ACL injury and surgery. All subjects in both groups had to meet the following inclusion criteria: 1) age between 14 and 50 years and 2) healthy, active individuals.

Exclusion criteria were: 1) Lower extremity injury/surgery in past 6 months other than ACL injury, 2) neurological disorders, and 3) neuromuscular disease. This study was approved by the Institutional Review Board of Mayo Clinic. Demographic information on the ACLR group and control group is shown in Table 1. Females in the ACLR group were significantly younger than those in control group. Other demographics did not demonstrate statistically significant differences between the control and ACLR group.

\subsection{Experimental Protocol}

For JPS testing, subjects were positioned and secured comfortably on a HumacNORM dynamometer (CSMi Technologies; Natick, MA; United States) in a seated position with the dynamometer arm secured around the distal shank $3 \mathrm{~cm}$ proximal to the lateral malleolus.

Subjects were blinded with the use of a blindfold placed over their eyes. The subjects were actively given three angles to reproduce $-15,45$, and 75 degrees from their anatomic zero (i.e. full extension of the knee) - in a randomized order. Each of the angles were reproduced actively by the subject three times after the initial angle position was locked by the HumacNORM with active extension of the subject's leg and the subject was requested to remember that joint position. The testing protocol was similar to the active-active JPS tests described previously with moderate to good reliability (Beynnon et al., 2000; Nagai et al., 2016). Each of the angles was performed with randomization of either localized vibration or no vibration. For a vibration trial, the stimulus would initiate after the subject had been given the opportunity to learn the angle but prior to commencement of reproducing the joint angle. The absolute angle differences between the reference position (target joint position) and replicated joint position was used as JPS (Beynnon et al., 2000). The average of three absolute angle differences was used for statistical analyses.

For JPS trials with vibration, localized vibration was supplied at $80 \mathrm{~Hz}$ frequency and 1.2 Vpp amplitude (sine waveform and burst mode) with an Arbitrary Waveform Generator voicecoil (Agilent 33220A; Agilent Technologies, Santa Clara, CA; United States). $80 \mathrm{~Hz}$ was selected as it has been shown to activate the majority of sensory fibers (especially muscle spindles) and also for its mid-range of demonstrated vibration effectiveness of the lower extremity (Bosco et al., 1999; Layne et al., 2014; Mano et al., 2006; Proske et al., 2014). The 1.2 Vpp was selected as it was the optimal amplitude to deliver maximal mechanical vibration. The air vibration was transferred via a 1 " diameter reinforced tubing and a rubber terminal for interface with the subject. The rubber terminal was positioned on the quadriceps tendon and secured with a Velcro strap around the distal femur/proximal to the patella (Figure 1). Joint angle data were collected on the HumacNORM interface software $(100 \mathrm{~Hz})$ with the computer technician recording the captured angle provided on the software when the subject verbally notified that they had reached the desired angle. 


\subsection{Statistical Analysis}

All statistical analyses were conducted with IBM SPSS statistical software Version 22 (IBM

Corp., Armonk, NY). Descriptive statistical analyses were used to calculate the means and standard deviations of JPS at each target position with and without vibration on the ACLR, contralateral, and control limbs. Both limbs of control subjects were tested; however, only the side that matched the ACLR limb in the ACLR group was chosen in the control group for analyses. The JPS differences between non-vibration and vibration trials were calculated by subtraction. A positive value reflects that JPS without vibration is larger than JPS with vibration. The normality was assessed with the Shapiro-Wilk test. One-way analysis of variance (ANOVA) or nonparametric equivalent (Kruskal-Wallis) was used to compare among limbs, followed by post-hoc analyses. Significance was set at $P<0.05$.

\section{Results}

Descriptive statistics of JPS at each angle with and without vibration are shown in Table 2. For all JPS trials without vibration, there were no significant differences among the ACLR, contralateral, and control limbs $(P<0.05)$. Similarly, for all JPS trials with vibration, there were no significant differences among the ACLR, contralateral, and control limbs $(P<0.05)$. When JPS differences between no-vibration and vibration trials were analyzed, there were no significant differences among the ACLR, contralateral, and control limbs $(P<0.05)$.

\section{Discussion}

The current investigation explored JPS with the quadriceps tendon vibration as a means to evaluate the integrity of the sensorimotor function in ACLR individuals. Overall, the JPS error values (both means and standard deviations) in the current investigation were within the ranges of JPS values reported in the previous studies (Angoules et al., 2011; Beynnon et al., 2000; Karasel et al., 2010; Nagai et al., 2016; Reider et al., 2003). This assured that the JPS protocols in the current investigation were properly executed. The ACLR limb was hypothesized to improve (smaller error values) with vibration while the contralateral and control limbs were hypothesized to decrease (larger error values) with vibration. Contrary to the hypothesis, there were no significant JPS differences with vibration.

Previously, authors investigated the effects of vibration on neck proprioception on individuals with and without neck pain, and reported significant improvement of neck JPS and postural sway with vibration in individuals with neck pain while individuals with no neck pain had worse neck JPS with vibration (Beinert et al., 2015). Others compared trunk JPS before and after vibration in healthy individuals and reported over a 1.5 times increase in JPS errors (Li et al., 2008). Although a different part of the body (neck vs. trunk vs. knee) was used to assess the effects of vibration on JPS, we anticipated at least some JPS changes in the control group. There were several potential reasons that could explain the current findings.

To the authors' knowledge, this was the first study to use vibration on the quadriceps tendon to study its effect on JPS in an ACLR group. Previous studies have indicated that vibrationinduced sensory changes could be muscle-specific. One study examined the sensory function 
using postural stability with and without vibration of three lower extremity muscles (triceps surae, quadriceps, and hamstrings) and reported significant postural alteration with vibration of the triceps surae while minimal changes were observed with vibration of quadriceps and hamstrings (Hirjakova et al., 2016). Similarly, another study reported that trunk and neck muscles were more sensitive to vibration and vibration-induced changes during walking and quiet stance than the lower extremity muscles (Courtine et al., 2007). Therefore, there may be issues with localization of vibration to the quadriceps tendon to induce adequate stimulation of the muscle spindle sensory fibers.

Unique to the quadriceps muscle is a large sesamoid bone (patella) that provides a mechanically advantageous lever arm between the muscle belly origins and the final insertion point on the tibial tuberosity (Grelsamer \& Weinstein, 2001). Consequently, especially with knee flexion, the patella and surrounding structures could act to absorb the applied vibration and divert the vibration stimulus from achieving adequate intensity to cause aberrant sensory information of the quadriceps musculature. Future studies should compare whether application of vibration to the tendon versus the quadriceps induces additional variance of error for JPS.

Second, vibration-induced post effect may have played a role in the current investigation. In other words, vibration-effect could have lasted longer and influenced "non-vibration" trials and JPS. Previous studies used the application of local vibration to the infrapatellar tendon for 20 minutes and compared the muscle strength and EMG before and after the vibration exposure (Konishi et al., 2002; Konishi, 2011). The current investigation re-introduced vibration each time subjects were assigned to either the vibration or non-vibration JPS, accordingly. This small difference in the order of vibration application could be a potential reason for a lack of significant findings. These vibration-induced post effects have been studied by assessment of postural stability repeatedly after vibration, and it was reported that application of 30-second vibration could negatively affect postural stability, and gradual return to baseline could take as long as 18 minutes post-vibration (Wierzbicka et al., 1998). This effect is likely related to the muscle spindle's thixotropic property (the dependence of a muscle's passive mechanical property on its previous history of contraction and length changes (Proske \& Gandevia, 2012)); both voluntary muscular contractions and vibration influence this property (Ishihara et al., 2004). Future studies with application of vibration should include assessment on separate days or design of the study to evaluate pre- and postvibration effects on JPS.

Another potential reason for no significant effects of vibration on JPS is the differences in vibration types: local versus whole-body vibration. In ACLR rehabilitation literature, wholebody vibration has been utilized as an exercise modality to help recruit additional motor units, especially early in ACLR rehabilitation (Rittweger, 2010). One clinical trial demonstrated that ACLR patients who went through conventional rehabilitation with wholebody vibration had better functional outcomes (hop test, shuttle run, isokinetic strength, and postural control) than the group without whole-body vibration, but not in JPS (Fu et al., 2013). While others found greater improvements in both postural stability and JPS with the whole-body vibration exercise group compared to the conventional exercise without vibration in the ACLR group (Moezy et al., 2008). The current investigation utilized 
localized vibration, not whole-body vibration. Given the effectiveness of the whole-body vibration as an exercise and therapeutic modality, it is of interest to determine whether whole-body vibration is a better modality to influence knee JPS than localized vibration.

Due to aforementioned reasons and potentially other factors, there were no significant JPS differences with and without vibration in the current study. However, it contributed to the body of literature in the sensorimotor function in the ACLR subjects. In the past, several investigations have been conducted to address the sensorimotor deficits in the ACLR subjects. A few studies focused on local or whole body vibration to increase quadriceps strength and rate of torque development in ACLR subjects (Konishi, 2011; Pamukoff et al., 2016). Other studies evaluated the changes/differences in electromechanical delay and cortical excitability between the ACLR and control groups and found that significant delay in the hamstring muscles during the maximum isometric contractions (Ristanis et al., 2009) and decreased cortical excitability (Pietrosimone et al., 2015). Due to high secondary injury rates, it is important to continue exploration for a simple yet comprehensive test that can be used to provide additional information when determining safe return-to-sport.

The current study had limitations. Although the subjects were blindfolded and the HumacNORM device is quiet in operation, the subjects may have utilized other senses to locate their joint position (i.e. sound) or relative position of the opposite limb that was not supplied with vibratory perturbation. Furthermore, the ACLR subjects were all cleared by their surgeon for sport participation and had undertaken intensive rehabilitation that likely emphasized joint sensory awareness with physical therapy. Accordingly, there may have been significant compensation of the sensorimotor system with integration of other proprioceptive systems. This was minimized with the subject seated in the HumacNORM and with vibratory perturbation to the quadriceps tendon as this would eliminate both vestibular and localized muscle spindle sensory inputs. However, sensory input from surrounding musculature and the contralateral joint may have been utilized for compensation.

\section{Conclusion}

The current investigation found minimal effects of localized vibration on JPS in the ACLR, contralateral, and control limbs. Several potential reasons such as vibration-induced post effect, locations of vibration, types of vibration, and rehabilitation status have been discussed. A refined means to assess individuals' sensorimotor system should be investigated. Future research should test ACLR subjects longitudinally with initial assessment within the time frame of injury and reconstruction and then observe sensorimotor changes over the course of rehabilitation, upwards to two years to ensure safe return-to-sport (Nagelli \& Hewett, 2017).

\section{Acknowledgments}

The authors would like to thank the subjects for their willingness to participate in the study. We acknowledge funding from the National Institute of Arthritis and Musculoskeletal and Skin Diseases: R01AR056259 and R01AR055563 to TEH and L30AR070273 to NDS, and from the National Institute of Child Health and Human Development: K12HD065987 to NDS. This work was also supported by the Kelly Orthopedic Fellowship, Mayo Clinic. 


\section{References}

Angoules AG, Mavrogenis AF, Dimitriou R, Karzis K, Drakoulakis E, Michos J, Papagelopoulos PJ. Knee proprioception following ACL reconstruction; a prospective trial comparing hamstrings with bone-patellar tendon-bone autograft. Knee. 2011; 18(2):76-82. [PubMed: 20149662]

Bates NA, Nesbitt RJ, Shearn JT, Myer GD, Hewett TE. Knee abduction affects greater magnitude of change in ACL and MCL strains than matched internal tibial rotation in vitro. Clin Orthop Relat Res. 2017 Epub ahead of print.

Beinert K, Keller M, Taube W. Neck muscle vibration can improve sensorimotor function in patients with neck pain. Spine J. 2015; 15(3):514-521. [PubMed: 25452010]

Beynnon, BD., Renstrom, P., Konradsen, L., Elmqvist, LG., Gottlieb, D., Dirks, M. Validation of techniques to measure knee proprioception. In: Lephart, SM., Fu, FH., editors. Proprioception and Neuromuscular Control in Joint Stability. Champaign, IL: Human Kinetics; -2000.p. 127-138.

Bonfim TR, Jansen Paccola CA, Barela JA. Proprioceptive and behavior impairments in individuals with anterior cruciate ligament reconstructed knees. Arch Phys Med Rehabil. 2003; 84(8):12171223. [PubMed: 12917863]

Bosco C, Tsarpela O. Influence of vibration on mechanical power and electromyogram activity in human arm flexor muscles. European Journal of Applied Physiology. 1999; 79:306-311.

Butler DL, Noyes FR, Grood ES. Ligamentous restraints to anterior-posterior drawer in the human knee. A biomechanical study. J Bone Joint Surg Am. 1980; 62(2):259-270. [PubMed: 7358757]

Claes S, Verdonk P, Forsyth R, Bellemans J. The "ligamentization" process in anterior cruciate ligament reconstruction: what happens to the human graft? A systematic review of the literature. Am J Sports Med. 2011; 39(11):2476-83. [PubMed: 21515806]

Courtine G, De Nunzio AM, Schmid M, Beretta MV, Schieppati M. Stance- and locomotion-dependent processing of vibration-induced proprioceptive inflow from multiple muscles in humans. $\mathrm{J}$ Neurophysiol. 2007; 97(1):772-779. [PubMed: 17065250]

Freeman MA, Wyke B. The innervation of the knee joint. An anatomical and histological study in the cat. J Anat. 1967; 101(Pt 3):505-532. [PubMed: 6051731]

Fu CL, Yung SH, Law KY, Leung KH, Lui PY, Siu HK, Chan KM. The effect of early whole-body vibration therapy on neuromuscular control after anterior cruciate ligament reconstruction: a randomized controlled trial. Am J Sports Med. 2013; 41(4):804-814. [PubMed: 23460328]

Goodwin GM, McCloskey DI, Matthews PB. The contribution of muscle afferents to kinaesthesia shown by vibration induced illusions of movement and by the effects of paralysing joint afferents. Brain. 1972; 95(4):705-748. [PubMed: 4265060]

Grelsamer RP, Weinstein CH. Applied biomechanics of the patella. Clin Orthop Relat Res. 2001; 389:9-14.

Hirjakova Z, Singliarova H, Bzduskova D, Kimijanova J, Buckova K, Valkovic P, Hlavacka F. Postural stability and responses to vibrations in patients after anterior cruciate ligament surgical reconstruction. Physiol Res. 2016; 65(Supplementum 3):S409-S416. [PubMed: 27775426]

Ishihara Y, Izumizaki M, Atsumi T, Homma I. Aftereffects of mechanical vibration and muscle contraction on limb position-sense. Muscle Nerve. 2004; 30(4):486-492. [PubMed: 15372539]

Iwasa J, Ochi M, Adachi N, Tobita M, Katsube K, Uchio Y. Proprioceptive improvement in knees with anterior cruciate ligament reconstruction. Clin Orthop Relat Res. 2000; 381:168-176.

Johansson H, Sjolander P, Sojka P. A sensory role for the cruciate ligaments. Clin Orthop Relat Res. $1991 ; 268: 161-178$.

Karasel S, Akpinar B, Gulbahar S, Baydar M, El O, Pinar H, Tatari H, Karaoglan O, Akalin E. Clinical and functional outcomes and proprioception after a modified accelerated rehabilitation program following anterior cruciate ligament reconstruction with patellar tendon autograft. Acta orthop Traumatol Turc. 2010; 44(3):220-228. [PubMed: 21088463]

Kim SH, Chun CH, Chun KC, Jo HJ, Kim KM. Histological assessment of mechanoreceptors in Achilles allografts after anterior cruciate ligament reconstruction. Am J Sports Med. 2012; 40(9): 2061-2065. [PubMed: 22837431] 
Konishi Y, Fukubayashi T, Takeshita D. Mechanism of quadriceps femoris muscle weakness in patients with anterior cruciate ligament reconstruction. Scand J Med Sci Sports. 2002; 12(6):371-375. [PubMed: 12453165]

Konishi YU. ACL repair might induce further abnormality of gamma loop in the intact side of the quadriceps femoris. Int J Sports Med. 2011; 32(4):292-296. [PubMed: 21380979]

Krogsgaard MR, Fischer-Rasmussen T, Dyhre-Poulsen P. Absence of sensory function in the reconstructed anterior cruciate ligament. J Electromyogr Kinesiol. 2011; 21(1):82-86. [PubMed: 21036626]

Layne CS, Chelette AM, Pourmoghaddam A. Impact of altered lower limb proprioception produced by tendon vibration on adaptation to split-belt treadmill walking. Somatosens Mot Res. 2014; 32(1): 31-38. [PubMed: 25162146]

Lehmann T, Paschen L, Baumeister J. Single-leg assessment of postural stability after anterior cruciate ligament injury: a systematic review and meta-analysis. Sports Med Open. 2017; 3(1):32. [PubMed: 28853022]

Li L, Lamis F, Wilson SE. Whole-body vibration alters proprioception in the trunk. Int J Ind Ergon. 2008; 38(9-10):792-800.

MacDonald PB, Hedden D, Pacin O, Sutherland K. Proprioception in anterior cruciate ligamentdeficient and reconstructed knees. Am J Sports Med. 1996; 24(6):774-778. [PubMed: 8947399]

Mano T, Iwase S, Toma S. Microneurography as a tool in clinical neurophysiology to investigate peripheral neural traffic in humans. Clin Neurophysiol. 2006; 117(11):2357-2384. [PubMed: 16904937]

Moezy A, Olyaei G, Hadian M, Razi M, Faghihzadeh S. A comparative study of whole body vibration training and conventional training on knee proprioception and postural stability after anterior cruciate ligament reconstruction. Br J Sports Med. 2008; 42(5):373-378. [PubMed: 18182623]

Nagai, T., Allison, KF., Schmitz, JL., Sell, TC., Lephart, SM. Sports Medicine. Dover, DE: SM Online Scientific Resources; 2016. Conscious proprioception assessments in sports medicine: how individuals perform each submodality?; p. 1-13.

Nagelli CV, Hewett TE. Should return to sport be delayed until 2 years after anterior cruciate ligament reconstruction? biological and functional considerations. Sports Med. 2017; 47(2):221-232. [PubMed: 27402457]

Nesbitt RJ, Herfat ST, Boguszewski DV, Engel AJ, Galloway MT, Shearn JT. Primary and secondary restraints of human and ovine knees for simulated in vivo gait kinematics. J Biomech. 2014; 47(9): 2022-2027. [PubMed: 24326097]

Ochi M, Iwasa J, Uchio Y, Adachi N, Kawasaki K. Induction of somatosensory evoked potentials by mechanical stimulation in reconstructed anterior cruciate ligaments. J Bone Joint Surg Br. 2002; 84(5):761-766. [PubMed: 12188501]

Ochi M, Iwasa J, Uchio Y, Adachi N, Sumen Y. The regeneration of sensory neurones in the reconstruction of the anterior cruciate ligament. J Bone Joint Surg Br. 1999; 81(5):902-906. [PubMed: 10530860]

Pamukoff DN, Pietrosimone B, Lewek MD, Ryan ED, Weinhold PS, Lee DR, Blackburn JT. Wholebody and local muscle vibration immediately improve quadriceps function in individuals with anterior cruciate ligament reconstruction. Arch Phys Med Rehabil. 2016; 97(7):1121-1129. [PubMed: 26869286]

Pietrosimone BG, Lepley AS, Ericksen HM, Clements A, Sohn DH, Gribble PA. Neural excitability alterations after anterior cruciate ligament reconstruction. J Athl Train. 2015; 50(6):665-674. [PubMed: 25844855]

Proske U, Gandevia SC. The proprioceptive senses: their roles in signaling body shape, body position and movement, and muscle force. Physiol Rev. 2012; 92(4):1651-1697. [PubMed: 23073629]

Proske U, Tsay A, Allen T. Muscle thixotropy as a tool in the study of proprioception. Exp Brain Res. 2014; 232(11):3397-3412. [PubMed: 25200179]

Reider B, Arcand MA, Diehl LH, Mroczek K, Abulencia A, Stroud CC, Palm M, Gilbertson J, Staszak P. Proprioception of the knee before and after anterior cruciate ligament reconstruction. Arthroscopy. 2003; 19(1):2-12. [PubMed: 12522394] 
Relph N, Herrington L, Tyson S. The effects of ACL injury on knee proprioception: a meta-analysis. Physiotherapy. 2014; 100(3):187-195. [PubMed: 24690442]

Ristanis S, Tsepis E, Giotis D, Stergiou N, Cerulli G, Georgoulis AD. Electromechanical delay of the knee flexor muscles is impaired after harvesting hamstring tendons for anterior cruciate ligament reconstruction. Am J Sports Med. 2009; 37(11):2179-2186. [PubMed: 19684295]

Rittweger J. Vibration as an exercise modality: how it may work, and what its potential might be. Eur J Appl Physiol. 2010; 108(5):877-904. [PubMed: 20012646]

Roberts D, Friden T, Stomberg A, Lindstrand A, Moritz U. Bilateral proprioceptive defects in patients with a unilateral anterior cruciate ligament reconstruction: a comparison between patients and healthy individuals. J Orthop Res. 2000; 18(4):565-571. [PubMed: 11052492]

Ruano JS, Sitler MR, Driban JB. Prevalence of radiographic knee osteoarthritis after anterior cruciate ligament reconstruction, with or without meniscectomy: An Evidence-Based Practice Article. J Athl Train. 2017; 52(6):606-609. [PubMed: 26930022]

Wierzbicka MM, Gilhodes JC, Roll JP. Vibration-induced postural posteffects. J Neurophysiol. 1998; 79(1):143-150. [PubMed: 9425185]

Young SW, Valladares RD, Loi F, Dragoo JL. Mechanoreceptor reinnervation of autografts versus allografts after anterior cruciate ligament reconstruction. Orthop J Sports Med. 2016; 4(10)

Zaffagnini S, De Pasquale V, Marchesini Reggiani L, Russo A, Agati P, Bacchelli B, Marcacci M. Neoligamentization process of BTPB used for ACL graft: histological evaluation from 6 months to 10 years. Knee. 2007; 14(2):87-93. [PubMed: 17188877] 


\section{Highlights}

- Anterior cruciate ligament injury can disrupt one's proprioception such as joint position sense.

- Sensory disruption is not likely restored through anterior cruciate ligament reconstruction.

- Localized vibration was used to examine sensorimotor function.

- With localized vibration, joint position sense did not differ among groups.

- Enhanced methodologies to assess sensory and motor function should be developed. 


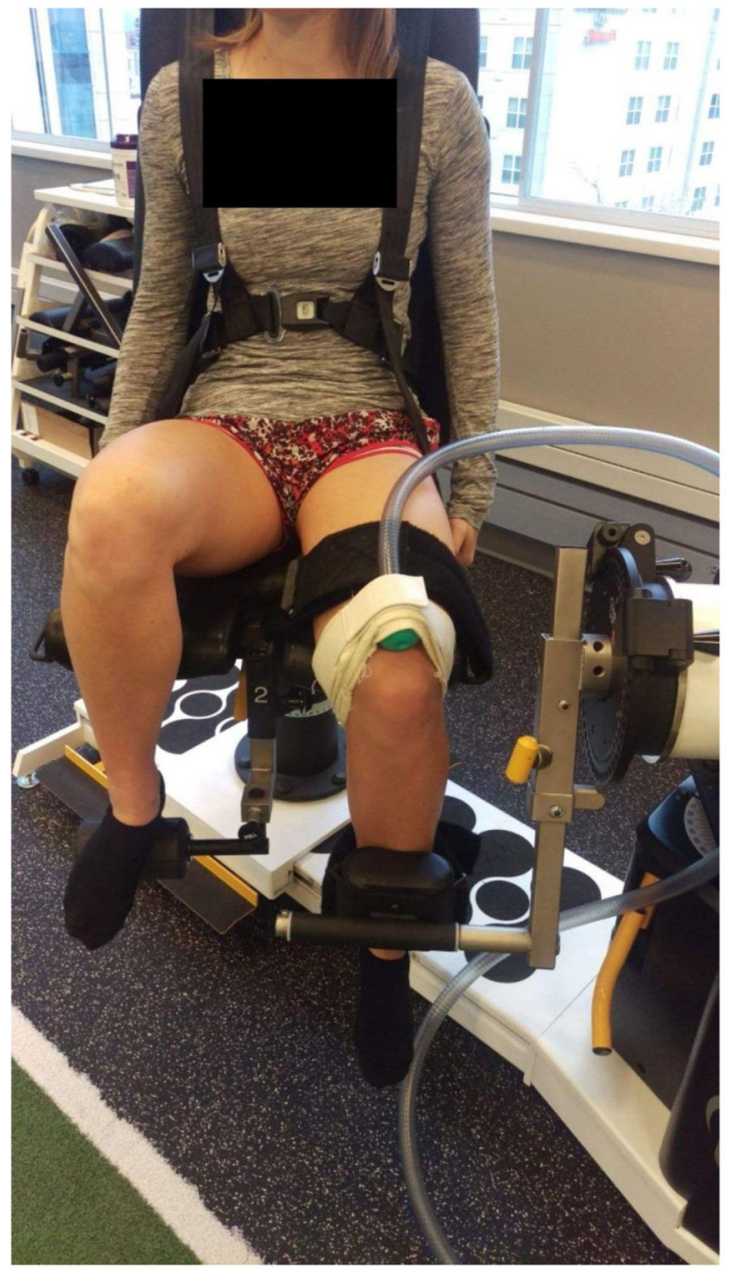

Figure 1.

Setup of vibration delivery to the quadriceps tendon. 


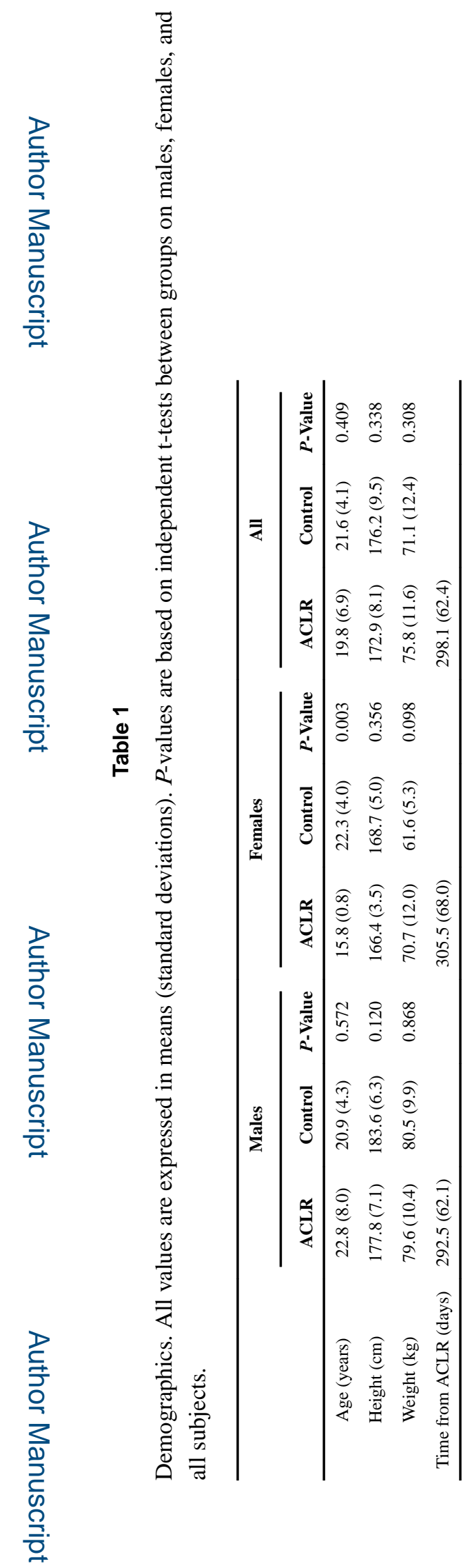

Clin Biomech (Bristol, Avon). Author manuscript; available in PMC 2019 June 01. 


\section{Table 2}

Comparisons of joint position sense absolute errors (in degrees) at target angles of 15, 45, and 75 degrees (or combined JPS of all angles) between the ACL-reconstructed (ACLR), contralateral, and control limbs with and without vibration. All values are means (standard deviations). $P$-values represent one-way analysis of variance or non-parametric equivalent (Kruskal-Wallis test).

\begin{tabular}{ccccc}
\hline No-Vibration & ACLR & Contralateral & Control & $P$-Value \\
\hline JPS 15 & $4.93(2.30)$ & $4.07(2.77)$ & $3.69(2.74)$ & $0.207^{*}$ \\
JPS 45 & $4.41(3.02)$ & $5.36(2.73)$ & $5.88(3.92)$ & $0.275^{*}$ \\
JPS 75 & $3.64(2.77)$ & $4.18(3.12)$ & $5.14(4.46)$ & $0.308^{*}$ \\
Combined & $4.24(1.59)$ & $4.44(1.99)$ & $4.91(2.69)$ & 0.431 \\
\hline Vibration & ACLR & Contralateral & Control & $P$-Value \\
\hline JPS 15 & $4.55(3.44)$ & $3.33(1.98)$ & $3.95(2.61)$ & $0.610^{*}$ \\
JPS 45 & $5.55(4.94)$ & $4.38(2.72)$ & $5.36(4.23)$ & $0.914^{*}$ \\
JPS 75 & $4.69(3.64)$ & $4.08(2.76)$ & $5.52(3.73)$ & $0.564^{*}$ \\
Combined & $4.82(2.50)$ & $3.83(1.72)$ & $4.94(2.49)$ & $0.894^{*}$ \\
\hline Differences & ACLR & Contralateral & Control & $P$-Value \\
\hline JPS 15 & $0.38(2.60)$ & $0.74(2.33)$ & $-0.26(3.10)$ & 0.557 \\
JPS 45 & $-1.14(3.77)$ & $0.98(2.29)$ & $0.52(4.34)$ & 0.288 \\
JPS 75 & $-1.05(4.71)$ & $0.10(3.08)$ & $-0.38(6.15)$ & $0.755^{*}$ \\
Combined & $-0.58(1.81)$ & $0.60(1.86)$ & $-0.04(3.22)$ & 0.590 \\
\hline * & & &
\end{tabular}

